\title{
Demokratisierung als Schlüssel für den Aufbau von Organisationsmacht: Erfahrungen des ver.di-Bezirks Stuttgart
}

\author{
JANA SEPPELT
}

Es ist in den letzten Monaten viel geschrieben worden über die Erfahrungen des ver.di-Bezirks Stuttgart mit Streiks und daraus folgender gewerkschaftlicher Erneuerung. ${ }^{1}$ Grund dafür ist, dass der Bezirk Stuttgart viel streikt, also eine hohe Anzahl von Streiktagen über teilweise lange Zeiträume hat. Grund dafür ist auch, dass Stuttgart erfolgreich streikt in dem Sinne, dass sich Beschäftigte aktiv einbringen, dass bisher nicht aktive Beschäftigtengruppen ${ }^{2}$ neu auftreten und eingebunden werden und durch demokratische Beteiligung eine Streikdynamik entsteht, die uns durchsetzungsfähiger macht. Und nicht zuletzt wurden wichtige Flächentarifauseinandersetzungen wie der Kampf um den Manteltarifvertrag 2007/08 im Einzelhandel, der Streik gegen die Einführung der 40-Stunde-Woche im öffentlichen Dienst (2005/2006) oder die letzte große Tarifauseinandersetzung 2013 im Einzelhandel hier in Baden-Württemberg mit in Stuttgart entschieden. Unsere Hauptthese ist, dass die Art und Weise, wie die Streiks in Stuttgart angelegt sind, die gewerkschaftliche Organisationsmacht ${ }^{3}$ steigert; dass also unser Fokus auf Demokratisierung des Streiks und wirkliche Beteiligung der Beschäftigten die Arbeitskampffähigkeit vergrößert.

Die Diskussion um gewerkschaftliche Erneuerung und die Steigerung gewerkschaftlicher Organisationsmacht ist aus zwei Gründen überlebensnotwendig für ver.di und andere Gewerkschaften: Zum einen werden Angriffe auf
Arbeitsbedingungen und Gehälter der Beschäftigten von Arbeitgeberseite offensiver geführt, gerade in Dienstleistungssektoren wie im Handel, aber auch im öffentlichen Dienst. Die Androhung einer Arbeitsniederlegung reicht bei Weitem nicht mehr aus, um die Arbeitgeberseite zu Zugeständnissen oder Kompromissen zu bewegen, sondern Beschäftigte müssen tatsächlich streiken, um ihren Tarifforderungen Nachdruck zu verleihen. Die Erfahrung im Dienstleistungsbereich ist, dass lange Auseinandersetzungen notwendig sind, um Verschlechterungen abzuwehren, von Verbesserungen ganz zu schweigen. Deutlich wird dies an der Tarifrunde Einzelhandel Baden Württemberg 2013. Ursprünglich als reine Entgeltrunde angelegt, entwickelte sich die Tarifauseinandersetzung nach der Kündigung des Manteltarifvertrages (MTV) durch den Arbeitgeberverband zu einem Konflikt um die Wiederinkraftsetzung des MTV. Die Arbeitgeberverbände hatten, um eine neue Entgeltstruktur zu „erpressen“, den MTV gekündigt. Hintergrund ist die Auseinandersetzung um eine neue Entgeltstruktur. Getarnt als „Modernisierung“ wollen die Arbeitgeber hier wesentliche Verschlechterungen bei der Eingruppierung und damit bei der Bezahlung u. a. der Kassiererinnen durchsetzen. Die Entgelterhöhung war vielen Streikenden zwar wichtig, trat aber in den Hintergrund, da die Verteidigung des MTV gegen die „Erpressungspolitik“ und die Zurückgewinnung der eigenen Handlungsfähigkeit vorrangig waren. Die Ausein-
1 Riexinger, B. (2011): Neue Streikkultur, in: LuXemburg 3 (4), S. 52-58; Schmalstieg, C. (2013): Partizipative Arbeitskämpfe, neue Streikformen, höhere Streikfähigkeit; Untersuchung der Gewerkschaftsarbeit des ver.di-Bezirks Stuttgart am Beispiel von Arbeitskämpfen im öffentlichen Dienst und derTarifrunde der Beschäftigten des Bundes und der Kommunen 2012: Analysen RosaLuxemburg-Stiftung.

2 U.a. weibliche Beschäftigte in Erziehungsberufen und im Einzelhandel sowie mehr junge Beschäftigte und Beschäftigte mit Migrationshintergrund, vgl. Riexinger, B. (2012), a. a. O. (Fußnote 1), S. 1.
3 Organisationsmacht entsteht durch den kollektiven Zusammenschluss der Beschäftigten in einer Organisation und wird im Jenaer Machtressourcenansatz abgegrenzt von der strukturellen Macht der Beschäftigten (Stellung im Produktionsprozess), der institutionellen Macht der Beschäftigten durch gesetzlich festgeschriebene Rechte und Institutionen sowie von kommunikativer bzw. gesellschaftlicher Macht (Beeinflussung gesamtgesellschaftlicher Diskurse/Öffentlichkeit), vgl. Schmalstieg, C. (2013), a.a. O. (Fußnote 1), S. 3; Dörre, K. (2014): Strategic unionism. Die Bedeutung von Streiks für gewerkschaftliche Erneuerung in Deutschland, in: Zeise, F./Hoffmann, R. (Hrsg.): Erneuerung durch Streik - die eigene Stärke nutzen, Beiträge aus Wissenschaft und Praxis, S. 5-13, S. 9. 
andersetzung dauerte über sieben Monate mit in der Spitze 104 Streiktagen in Stuttgart.

Zum anderen ist im Dienstleistungssektor die Organisationsmacht häufig der entscheidende Machtfaktor, da ein direkter ökonomischer Schaden durch die Niederlegung der Produktion selten entsteht. Nun ist die Macht durch den kollektiven Zusammenschluss innerhalb von ver.di sehr unterschiedlich ausgeprägt, da Mitglieder eben nicht automatisch aktive Mitglieder sind und mancherorts gar keine Mitglieder vorhanden sind. Organizing-Ansätze sind noch nicht genügend in die Praxis integriert und Streikstrategien häufig nicht an die Gegebenheiten des Sektors und die Dynamik der Auseinandersetzung angepasst.

Wir stehen innerhalb der Organisation erst am Anfang dieser wichtigen Diskussion und Bewegung: Basisaufbau und Organizing, das sinnvolle Arbeiten mit Betriebsatlanten/Mapping ${ }^{4}$ oder ein gemeinsamer Erfahrungsaustausch über Erfolge sind keine Selbstverständlichkeiten. Was für Organizing als Praxis eines Basisaufbaus im Betrieb und einer Demokratisierung der Entscheidungsstrukturen gilt, gilt auch für die demokratische Streikpraxis innerhalb der Gesamtorganisation: Die schwerste Arbeit liegt noch vor uns. ${ }^{5}$ Die Erfahrungen erfolgreicher Bezirke sollten mehr verallgemeinert werden anstatt bei business as usual zu bleiben. In Flächentarifauseinandersetzungen wie im öffentlichen Dienst 2012 verhindert ein Ungleichgewicht zwischen den Bezirken nicht selten tarifpolitische Erfolge. Ver.di Stuttgart hat im letzten Jahr einen ersten Schritt für den Austausch der Erfolge und ihrer Voraussetzungen getan und im Rahmen der Konferenz „Erneuerung durch Streik“ gemeinsam mit der Rosa-Luxemburg-Stiftung eben diesen Austausch organisiert. Vom 2. - 4. Oktober diesen Jahres ist die nächste Konferenz geplant, die der ver.di-Bezirk Hannover zusammen mit der IG Metall Hannover und der Rosa-Luxemburg-Stiftung organisiert.

\section{Neue (Streik)Kultur und Demokratisierung}

Sollen betriebliche Auseinandersetzungen oder Streiks erfolgreich sein, verlangt das eine hohe Einbindung der Beschäftigten in die Auseinandersetzung. Nun ist die Ausgangslage in der Dienstleistungsbranche nicht selten, dass wir einen relativ niedrigen Organisationsgrad vorfinden (wie im öffentlichen Dienst) und es unter den Beschäftigten eine hohe Fluktuation gibt (wie beispielsweise im Einzelhandel). Es klingt einfach, war und ist aber voraussetzungsvoll: Für eine Forderung oder mehrere Forderungen aktiv zu werden, erfordert für Beschäftigte unter anderem, dass sie die Forderung teilen; dass sie bereit sind, sie aktiv zu vertreten und dass sie sich trauen, dafür auch im Betrieb die Arbeit zu verweigern und für ihre Belange auch vor Kolleginnen und Kollegen einzustehen. Leichter fällt ihnen das, wenn sie kollektiv handeln, also in der Auseinandersetzung nicht allein (gelassen) sind und die Unterstützung der Öffentlichkeit und der Nutzerinnen und Nutzer der Dienstleistung für ihre Forderungen gewinnen. Haupt- und
Ehrenamtliche sollten das im Hinterkopf behalten, die Vorbereitung und Durchführung des Streiks sowie interne Abläufe daran anpassen und sich auf die Auseinandersetzung und den Streik als Prozess einlassen.

\section{Aktiver Kern und breite Forderungsaufstellung}

Ohne einen aktiven Kern an Ehrenamtlichen gibt es keine basisnahe betriebliche und tarifliche Politik. Im öffentlichen Dienst stellt die ehrenamtliche Streikleitung den Kern: In ihr sitzen die Vertreter und Vertreterinnen der Betriebe (insbesondere der Streikbetriebe) und entscheiden gemeinsam mit den Hauptamtlichen über die Vorgehensweise und Streikstrategie. Die Forderungen werden von Anfang an breit auf Mitgliederversammlungen diskutiert und zurück an die Streikleitung kommuniziert. Neben den Forderungen wird in der Streikleitung schon früh über mögliche Streikstrategien nachgedacht; auch in den Mitgliederversammlungen wird darüber geredet, wie ein Streik aussehen wird. Erzieher und Erzieherinnen beispielsweise fragen früh, wie man denn mit den Eltern umgeht oder ob man mit einer langen Auseinandersetzung zu rechnen hat. Voraussetzung für ein Gelingen der Diskussion ist eine Kommunikation auf Augenhöhe, in der die Ansichten oder Bedenken der Beschäftigten gehört und diskutiert werden. Dies beinhaltet die Entwicklung einer gewissen Konflikt- und Streitfähigkeit, das Vorhandensein von Ressourcen (Zeit) und die Fähigkeit (auch von hauptamtlicher Seite), eigene Positionen zur Diskussion zu stellen und anzupassen. 2006 streikten die Beschäftigten des öffentlichen Dienstes in Stuttgart neun Wochen lang in unterschiedlicher Intensität. Dies war einer Streikstrategie zu verdanken, die in täglichen Streikleitungssitzungen, die offen für alle waren, immer an die aktuellen Geschehnisse angepasst worden ist. Nach langen Diskussionen führte dies dazu, dass für den Bereich der Abfallwirtschaft eine Strategie entwickelt wurde, die für die Arbeitgeberseite nicht mehr kalkulierbar war. Im Ergebnis konnte damit der Einsatz von privaten Abfallwirtschaftsunternehmen unterlaufen werden. Dadurch entstanden der Landeshauptstadt Stuttgart enorme Kosten. Diese Strategie wurde mittlerweile auch von den Beschäftigten des Einzelhandels in den Streiks 2007/08 und 2013 übernommen. Sie ist deshalb so erfolgreich, da sie auf einer breiten Basis aufbaut, von allen mitgetragen wird und natürlich in je-

4 Mapping heißt übersetzt so viel wie "abbilden" oder "kartieren“. Von Betrieben oder Arbeitsbereichen wird eine "Landkarte" erstellt, die die Betriebsstruktur sowie die Anzahl der Beschäftigten und gewerkschaftlich Organisierten in den Abteilungen und Bereichen aufzeigt, vgl. Karakayali, J. (2009): Organisiert euch! Handbuch für Unternehmensrecherche, Eigendruck des ver.di Bundesvorstands, S. 67.

5 Zu Organizing vgl. Raffo, J. (2014): Die schwerste Arbeit liegt noch vor uns! Ein Gespräch mit Jeffrey Raffo über Organizing in Deutschland, in: express 01/2014. 
dem Fall gemeinsam diskutiert und umgesetzt werden muss. Verordnen lassen sich derartige flexible Streikstrategien nicht: Ohne breite Basis und Rückhalt im Betrieb ist es schwer und unangenehm, immer wieder die Arbeit aufzunehmen und dort als Streikende beschimpft oder gemobbt zu werden. Damit flexible Strategien erfolgreich sind, müssen diese großflächig im Betrieb getragen werden.

\section{Kampagnenförmige Auseinandersetzung und Öffentlichkeitsarbeit}

Um zu erreichen, dass sich auch nicht organisierte Beschäftigte mit den Forderungen identifizieren, aktiv werden und beteiligen, muss die Diskussion um Forderungen früh in die Betriebe getragen werden. In den Streiks im Erziehungsbereich wurden im Vorfeld des Arbeitskampfes nicht nur die Stuttgarter Erziehungseinrichtungen von gewerkschaftlich organisierten Personalräten und Vertrauensleuten besucht, sondern auch zahlreiche Erziehungseinrichtungen in den Landkreisen (Böblingen, Ludwigsburg und RemsMurr). Das sollte sich als richtiger Schritt herausstellen: Die Streikbeteiligung war nicht nur wie gewohnt in der Landeshauptstadt hoch, sondern zum ersten Mal auch in den Landkreisen. Insbesondere in den Kreisstädten, aber auch in einigen kleineren Städten und Gemeinden, kam es zu ähnlich intensiven Streiks wie in Stuttgart. ${ }^{6}$

Durch haupt- und ehrenamtliche Gewerkschafter wurden die Kitas in Städten und Gemeinden ohne funktionierende Vertrauensleutestrukturen besucht. Der direkte, persönliche Kontakt in die Kita hinein machte den Erfolg der Streikbewegungen 2009 und 2012 aus. Die Besuche sind keine einmalige Angelegenheit geblieben, sondern wurden in regelmäßigen Abständen wiederholt, teilweise sind Netzwerke und Verbindungen entstanden, die auch unabhängig von den Tarifbewegungen tragen. In der Folge dieser Besuche entwickelten wir mit den Ehrenamtlichen fachlich auf Kitas zugeschnittene Angebote, die zum einen an der Bildungsarbeit ansetzten, aber auch die Rahmenbedingungen für eine gelingende Bildungsarbeit bearbeiteten. Diese Vorfeldarbeit, d.h. „das Abholen der Beschäftigten in ihrer Fachlichkeit" ist unabdingbar für den Erfolg einer Tarifkampagne. Ein Beispiel: Der Grundsatz im pädagogischen Bereich, dass die Bildungsarbeit nur mit guten Arbeitsbedingungen und ohne Fachkräftemangel zu bewältigen ist, knüpft direkt am beruflichen Alltag der Erzieherinnen und Erzieher und den sich hier stellenden Problemen an. Der Streik 2009 hat gezeigt, dass auch Unorganisierte bereit sind, mitzumachen, wenn in den Forderungen ein Bezug zur beruflichen Alltagsrealität hergestellt wird.

Wichtig ist des Weiteren, dass die Diskussion in gesellschaftliche Auseinandersetzungen eingebettet wird. In der Tarifauseinandersetzung 2012 haben wir im Bezirk eine Kampagne zur Verbreiterung der Forderungen und ihrer Legitimierung in der Krise aufgelegt. Diese stand unter dem Motto „Die nächsten Milliarden für uns“ und bestand aus vier Flugblättern, die offensiv die Verwendung öffentlicher
Mittel für die Bankenrettung und die verfehlte Steuerpolitik kritisierte. Statt dem neoliberalen Credo „Privater Reichtum und öffentliche Armut" zu folgen, solle die öffentliche Hand lieber gute Arbeit finanzieren und ausgelagerte Bereiche zurückholen. Über die Eintrittserklärungen auf den Flugblättern kamen in Stuttgart im Vorfeld neue Gewerkschaftsmitglieder dazu. Gleichzeitig haben wir früh die Presse mit ähnlichen Argumenten versorgt - mit dem Ergebnis, dass die Medien in der Auseinandersetzung auf unserer Seite standen.

Gerade im Dienstleistungsbereich müssen Nutzer und Kunden früh mit in die Auseinandersetzung einbezogen werden. Dabei ist zentral, zu vermitteln, für welche Forderungen gestreikt wird und welche Auswirkungen Verbesserungen der Arbeitsbedingungen auf die Nutzer haben. Im öffentlichen Dienst ist das umso wichtiger: Zum einen ist dieser u. a. aus den Steuern der Bürger und Bürgerinnen finanziert, zum anderen werden durch Streiks notwendige öffentliche Dienstleistungen (Verkehr, Krankenhaus, Kitas) beeinträchtigt. Zur Streikpraxis in Stuttgart gehört es beispielsweise, schon früh Eltern über Elternbriefe und Treffen mit den Elternbeiräten mit einzubeziehen.

Werden Bedürfnisse der vom Streik Betroffenen mit bedacht und wird kreativ mit diesen zusammen nachgedacht, gelingt hin und wieder auch eine Streikstrategie wie bei den Stuttgarter Straßenbahnen: Durch das Bestreiken der Fahrkartenverkaufsstellen und des Servicepersonals sowie der Kontrolleure hatten die Stuttgarter freie Fahrt und der Arbeitgeber trotzdem ökonomischen Schaden. ${ }^{7}$ Selbiges gilt auch für den Streik des Küchenpersonals in den Stuttgarter Kitas. Da nur das Küchenpersonal streikte, musste die Verpflegung der Kinder extern organisiert werden. Die Eltern haben vertraglich ein Recht auf die Verpflegung ihrer Kinder, so war der Schaden an dieser Stelle für die Stadt Stuttgart heftig.

\section{Gemeinsame Entscheidungen während des Streiks: Die Rolle der Streikversammlung}

In Stuttgart ist es Praxis, an jedem Streiktag eine Streikversammlung im Gewerkschaftshaus zu machen, auf der die Forderungen und Strategien der Arbeitgeberseite diskutiert werden. Ausnahmen sind sogenannte stille Streiks, z. B. am Wochenende oder an Brückentagen, wenn Erholungsphasen sinnvoll sind. Die kollektive Erfahrung der rein zahlenmäßigen Menge und die Diskussion stärken nicht nur die Streikenden, es entwickeln sich auch aus der Situation eine ganz

6 Riexinger (2012), a. a. O.

7 Siehe auch Höpfner, W. (2014): Glück gehört dazu, Neue strategische Ansätze beim Streik 2012 im öffentlichen Personennahverkehr in Baden-Württemberg; in Zeise, F./Hoffmann, R. (Hrsg.): Erneuerung durch Streik - die eigene Stärke nutzen, Beiträge aus Wissenschaft und Praxis, S. 18-20. 
eigene Dynamik und kreative Aktionsformen. Die Hauptamtlichen haben eine mobilisierende wie moderierende Rolle. Sie sind Teil des Streiks und treiben die Sache voran. Das gelingt nur, wenn sie das Vertrauen der Streikenden haben und das haben sie nur, wenn von Anfang an eine wirkliche Beteiligung stattfindet.

Neben der Strategiediskussion, dem Erfahrungsaustausch, aber auch der Kritik an organisatorischen Abläufen werden kreative Aktionen diskutiert, geplant und umgesetzt. Entstanden sind bei solchen Aktionen große Performances, die mit mehreren Tausend Streikenden durchgeführt wurden. Entwickelt wurden aber auch viele kleine Ideen, die einem Flashmob nahekommen: Vom „Besuch des Rathauses“ durch streikende Beschäftigte im öffentlichen Dienst über Formen des „kreativen Einkaufens“; die Einbeziehung der Öffentlichkeit durch „Gutscheine“8 ${ }^{\text {" }}$ bis hin zu betriebsbezogenen Aktionen wie z. B. das Lahmlegen der „Präsentation und Einkaufsmöglichkeit von Designerbekleidung“9 Besonders zu erwähnen ist die Blockade des Müllheizkraftwerkes durch streikende Müllwerker, nach dem der öffentliche Arbeitgeber private Unternehmer zur Abholung des Mülls eingesetzt hatte. Diese Aktion wurde auf einer frühmorgendlichen Streikversammlung gemeinsam erörtert. Umgehend umgesetzt hatte sie für den Streik damals eine enorm stabilisierende Wirkung und der Schaden für den Arbeitgeber war enorm.

Wie schon in der Forderungsdiskussion ist auch während des Streiks das Austragen von Konflikten zentral für das Gelingen der Auseinandersetzung. Durch die unterschiedliche Mobilisierungskraft entstehen Widersprüche, so treffen beispielsweise die, die am liebsten sofort und unbegrenzt weiter streiken wollen, auf zögerliche Kolleginnen und Kollegen oder jene, die unter schlechteren Bedingungen beschäftigt sind. Derartige Interessengegensätze müssen dann ausgetragen und zu einem Ergebnis geführt werden, das die einen nicht bremst und die anderen mitnimmt. Der Streik und die Streikplanung werden so zu einer Sache aller Streikenden. Das stärkt die Kampfkraft, die Verbindlichkeit und die Identifikation mit der Gewerkschaft. ${ }^{10}$

\section{Gute Nachbereitung ist so wichtig wie gute Vorbereitung}

Auch nach einer Auseinandersetzung, nach einem Streik, wird das Ergebnis auf Streikversammlungen diskutiert. Wir hatten durch die oben schon erwähnten Ungleichgewichte in der Organisationsmacht innerhalb der Organisation gerade im öffentlichen Dienst die Situation, dass der ver.diBezirk Stuttgart gegen das Tarifergebnis stimmte (mit $54 \%$ aller Mitglieder), der Großteil der Organisation allerdings dafür. Das ist gerade für die Aktiven nicht einfach zu verdauen. Zur Nachbereitung mit den Ehrenamtlichen gehört an diesem Punkt die Herstellung von Transparenz über Verlauf und Inhalte der Verhandlungen und, wenn nötig, auch interne Kritik und Intervention. Viele interne Kritikpunkte an der Tarifrunde 2012, wie beispielsweise eine längere Aktivierungsphase und politisch offensivere Öffentlichkeitsarbeit, wurden 2014 zumindest teilweise berücksichtigt.

Allerdings ist die Geduld endlich, früher oder später muss das Problem geografisch unterschiedlicher Mobilisierungsfähigkeit angegangen werden, um die Beschäftigten in den Streikhochburgen nicht über kurz oder lang zu frustrieren. Wir müssen in und außerhalb von ver.di Räume schaffen, in denen wir voneinander lernen können. Veranstaltungen wie die Streikkonferenz im März 2013 in Stuttgart und die kommende Streikkonferenz im Herbst 2014 in Hannover können hier Abhilfe schaffen, ersetzen aber nicht die innerorganisatorische Verallgemeinerung erfolgreicher Strategien.

\section{AUTORIN}

JANA SEPPELT, Politikwissenschaftlerin, Gewerkschaftssekretärin für den Fachbereich 7 beim ver.di-Bezirk Stuttgart.

jana.seppelt@verdi.de

8 Unterstützer der Streikenden haben im Einzelhandel „Gutscheine" an Kunden verteilt, die zu "50 \% Personalkostenreduzierungen auf allen Ebenen berechtigten“. Im Schwabenländle führte das dazu, dass die Kundschaft „Rabatt" las, die Streikbrecher wurden dem Ansturm nicht mehr Herr und in den "beteiligten Modestores" brach das Chaos aus.

9 Ein bekannter Modestore eröffnet der Kundschaft zeitlich begrenzt vor Ladenöffnung die Möglichkeiten, Kleidung, die von einer bekannten Designerin entworfen wurde, zu kaufen. Der Laden blieb leer, da sich massenweise Unterstützer als normale Kunden Zugangsberechtigung verschaffen konnten.

10 Riexinger (2012), a. a. O. 